\title{
Microfluidic Push-Pull Probe for Scanning Electrochemical Microscopy
}

\author{
Dmitry Momotenko, ${ }^{\dagger}$ Fernando Cortes-Salazar, ${ }^{\dagger}$ Andreas Lesch, ${ }^{\ddagger}$ Gunther Wittstock, ${ }^{\ddagger}$ and \\ Hubert H. Girault*, ${ }^{*}$ \\ ${ }^{\dagger}$ Laboratoire d’Electrochimie Physique et Analytique, Ecole Polytechnique Fédérale de Lausanne, Station 6, \\ CH-1015 Lausanne, Switzerland \\ ${ }^{\ddagger}$ Department of Pure and Applied Chemistry, Center of Interface Science, Faculty of Mathematics and Natural Sciences, \\ Carl von Ossietzky University of Oldenburg, D-26111 Oldenburg, Germany
}

Supporting Information

ABSTRACT: This paper presents a microfluidic push-pull probe for scanning electrochemical microscopy (SECM) consisting of a working microelectrode, an integrated counter/reference electrode and two microchannels for pushing and pulling an electrolyte solution to and away from a substrate. With such a configuration, a droplet of a permanently renewed redox mediator solution is maintained just at the probe tip to carry out SECM measurements on initially dry substrates or in microenvironments. For SECM imaging purposes, the probe fabricated in a soft polymer material is used in a contact regime. SECM images of various gold-on-glass samples demonstrate the proof-of-concept of a push-pull probe for local surface activity characterization with high spatial resolution even on vertically oriented substrates. Finite element computations were performed to guide the

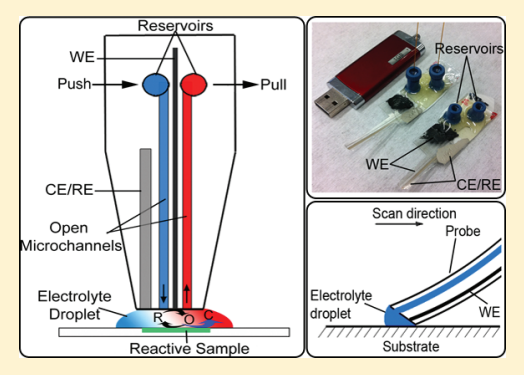
improvement of the probe sensitivity.

$\mathrm{T}$ he recent miniaturization trend in science and technology requires new tools for micro- and nanometer-scale characterization of surfaces with a high spatial resolution; for example, to monitor the stability of coatings and to characterize nanostructures, biological membranes, tissues, and even living cells. ${ }^{1}$ In most cases, optical microscopy is the primary detection tool; however, this technique is unable to provide chemical or electrochemical information. Scanning electrochemical microscopy (SECM) is a scanning probe microscope technique to characterize surface reactivity at almost any interface, e.g., liquid-liquid, liquid-solid, and liquid-gas. ${ }^{2-12}$ SECM setup is normally accomplished with a probe ultramicroelectrode (UME), a positioning system in $x, y$, and $z$ directions, and a (bi)potentiostat to control the potential and to measure the amperometric response of the probe. When electrochemical reactions (i.e., reduction or oxidation of a redox mediator) occur at the UME, a steady-state diffusion-limited electrical current can be established. The magnitude of this probe response strongly depends on the probe-substrate distance $d$ and the electrochemical reactivity of the sample under study. Therefore, by moving the probe in $x$ and $y$ directions, one can map the chemical reactivity of a particular interface ${ }^{13-18}$ and extract kinetic data by comparison with numerical simulations of mass transport and interfacial kinetics in the interelectrode space. ${ }^{19-25}$

A major challenge for SECM is the study of real systems, like inspection of coatings on mechanical pieces that cannot be taken out for investigation, corrosion studies, and scanning of human fingerprints on various substrates in forensic sciences, because immersing large, bent, or delicate substrates into an electrolyte solution is often cumbersome. In order to perform SECM experiments in such conditions, different approaches have been proposed, ranging from working inside a thin layer of water (i.e., a few nanometers or even less) ${ }^{26,27}$ to the use of an ultrasmall electrolyte volume (i.e., $\sim \mathrm{nL}$ ) located just between the sample and the probe. In the latter case only a small part of the sample surface is in contact with the electrolyte solution, usually for a short period of time that is particularly convenient for systems where corrosion, surface passivation, or fouling occur. The difficulties present in such a small-scale configuration arise from electrode collision, solvent evaporation, and surface wetting. Different approaches, such as microcapillary-based techniques like scanning droplet cell ${ }^{28-30}$ or scanning micropipet contact method $^{31}$ have been successfully employed for surface reactivity characterization on different substrates. Usually, the setup is an integrated two- or three-electrode cell where the surface of the working electrode is defined by the area of the electrolyte droplet delivered from the capillary. Electrochemical imaging is thus implemented by moving the droplet across the substrate by displacing the probe or the sample. The major limitation of such configuration is the fact that only conductive samples can be studied, restricting the application scope. Recently, a scanning electrochemical cell microscopy has been reported that

Received: March 16, 2011

Accepted: May 12, 2011

Published: May 12, 2011 
utilizes a theta pipet electrochemical cell for simultaneous conductance and topographical imaging of conductive and semiconductor substrates. ${ }^{32}$ The operation principle is based on the periodic deformation of the liquid meniscus that modulates the solution resistance and consequently produces independent direct (dc) and alternating (ac) current components for functional surface imaging.

Another strategy for performing SECM experiments in microand nanoenvironments is based on measurements within a stationary droplet of electrolyte solution. ${ }^{33,34}$ Spaine and Baur ${ }^{33}$ reported a positionable two-electrode microcell system localized in a theta pipet, where one channel of the pulled theta glass capillary contains a carbon fiber sealed in epoxy resin, while the other one includes an $\mathrm{Ag} / \mathrm{AgCl}$ reference electrode separated from the external electrolyte solution by an agar salt bridge. Successful SECM imaging of a Pt disk electrode (10 $\mu \mathrm{m}$ diameter) was achieved in a picoliter droplet by controlling solvent evaporation with a humidity chamber. Since solvent evaporation becomes a critical condition for image acquisition with SECM, different strategies have been employed for overcoming such situations, for example, by adding glycerin to the electrolyte or covering the solution with mineral or paraffin oil. ${ }^{34-38}$ In the latter example, ${ }^{34}$ a coaxial two-electrode configuration was built by depositing a layer of metallic silver on the glass body that encloses the Pt microdisc electrode. SECM images in feedback mode of $\mathrm{Pt}$ bands were achieved without presenting a drastic solvent evaporation influence. However, working with stationary electrolyte droplets restricts the size of the scanning area to that of the droplet, and attention has to be paid in order to avoid sample contamination with the covering oil phase. An additional drawback of the approaches mentioned above is the fact that upon contact between the capillary or the glass microelectrode and the substrate, destruction of the sample or the fragile probe may occur. The latter is more relevant when scanning rough, tilted, and curved substrates where topographical artifacts can be introduced by sporadic and nonuniform changes on $d$. To alleviate the latter problem, an additional feedback loop for current-independent distance control can be introduced to the SECM setup. In such cases SECM is coupled to a shear forcebased distance control with optical, ${ }^{39,40}$ piezoelectric, ${ }^{41}$ or tuning fork-based detection ${ }^{42,43}$ of shear forces, scanning ion conductance microscopy, ${ }^{44,45}$ tip impedance-based feedback system, ${ }^{46,47}$ ac perturbation of the probe position, ${ }^{48}$ attenuated total reflection infrared spectroscopy (IR-ATR), ${ }^{49}$ and force detection by atomic force microscopy (AFM). ${ }^{50-54}$

Recently reported soft stylus probes ${ }^{55}$ and soft microfabricated arrays ${ }^{56}$ provide a simple approach for electrochemical activity mapping on curved, tilted, corrugated, and large substrates. Soft probes are scanned in contact mode (similar to in AFM) maintaining a constant $d$, since the soft material of the electrode can accommodate the surface topography of the sample. As shown recently, ${ }^{57}$ a two-electrode cell coupled with a fountain pen-type microfluidic system to deliver a nanoliter droplet of redox mediator solution to the soft probe tip is an effective way to probe surface activity on initially dry surfaces, while taking advantage of the SECM measurements in contact mode. However, sample contamination after scanning remained a major issue that can affect not only the sample but also the imaging process if supporting electrolyte precipitates and changes the bending degree of the fountain pen probe.

Herein, we report a push-pull probe for surface reactivity characterization that integrates a working electrode (WE), a counter/reference electrode (CE/RE), and a microfluidic system. Similar to the multipurpose microfluidic probe reported by Juncker et al. $^{58}$ for working in solution bulk or in microenvironments, ${ }^{59}$ this probe contains two microchannels for delivery and aspiration of redox mediator solution at the probe tip. Such configuration is designed to conduct SECM measurements within a constantly renewed nanodroplet of electrolyte, avoiding significant solvent evaporation effects, such as sample contamination or increasing of redox mediator concentration. Push-pull probes encompass the advantages of soft probes (i.e., scanning over tilted and/or rugged substrates) and circumvent the principal drawback of single-channel microfluidic fountain pen probes. The capability for surface reactivity detection on vertical substrates with minimal changes in SECM setup is shown, in addition to a theoretical study for probe sensitivity improvement when working in SECM contact mode.

\section{EXPERIMENTAL SECTION}

Chemicals. Ferrocene methanol $\left(\mathrm{FcCH}_{2} \mathrm{OH}, \geq 97 \%\right.$, SigmaAldrich) and $\mathrm{KNO}_{3}$ ( $\geq 99 \%$, Buchs, Switzerland) were used as received. Deionized water was produced by a Milli-Q plus 185 model from Millipore (Zug, Switzerland). Push-pull probes were fabricated by use of $100 \mu \mathrm{m}$ thick poly(ethylene terephthalate), Melinex (PET; Dupont, Wilmington, DE) and $50 \mu \mathrm{m}$ polyethylene/poly(ethylene terephthalate) (PE/PET; Payne, Banbury, England) lamination foils. Laser-machined tracks in PET were filled by Electrador carbon ink (Electra Polymer \& Chemicals Ltd., Dunk Green, England) for the working electrode and by $\mathrm{Ag} / \mathrm{AgCl}$ ink (ERCON, Wareham, MA) for the CE/RE electrode.

Preparation of Gold Printed EPFL Logo and Gold Microelectrode Arrays. Glass slides were treated with piranha solution and cleaned by sonication in deionized water followed by drying under a stream of argon. Caution: This mixture reacts violently with all organic material. Piranha solution has to be handled with extreme care to avoid personnel injury and property damage. Microchips with gold printed EPFL logo and interdigitated array of electrodes, $100 \mathrm{~nm}$ thick, were prepared in an Edwards Auto 306 evaporator operating at a pressure less than $5 \times 10^{-6}$ mbar. A metallic mask defined the microstructure shape. The film growth was initiated by the thermal evaporation of a $1 \mathrm{~nm}$ thick chromium (99.99\%, Balzers) layer at $<0.1 \mathrm{~nm} / \mathrm{s}$ in order to enhance the adhesion of the Au layer. Gold (99.99\%, Balzers) was subsequently evaporated at $<0.1 \mathrm{~nm} / \mathrm{s}$ up to $5 \mathrm{~nm}$ before the deposition rate was increased to $0.2-0.3 \mathrm{~nm} / \mathrm{s}$ for the deposition of a $100 \mathrm{~nm}$ layer.

Push-Pull Probe Preparation. The open microchannels for the microfluidic system, the working electrode (WE), and the counter/reference electrode (CE/RE) were ablated in a PET film of $100 \mu \mathrm{m}$ through metallic masks by use of a $193 \mathrm{~nm}$ ArF excimer laser beam (Lambda Physik, Göttingen, Germany, fluence $=0.2 \mathrm{~J}$, frequency $=50 \mathrm{~Hz}$ ) as reported previously. ${ }^{55-57,60,61}$ Microchannels with $60 \mu \mathrm{m}$ width, $40 \mu \mathrm{m}$ depth, and $6.3-6.8 \mathrm{~cm}$ length were positioned on one side of the PET film, while on the opposite side the microchannels for $\mathrm{WE}$ ( $55 \mu \mathrm{m}$ width, $30 \mu \mathrm{m}$ depth, and $5 \mathrm{~cm}$ length) and CE/RE (1 mm width, $20 \mu \mathrm{m}$ depth, and $4 \mathrm{~cm}$ length) were also aligned. The WE microchannel was centered with respect to the position of the two open microchannels that are separated by $100 \mu \mathrm{m}$, while CE/RE was placed $2 \mathrm{~mm}$ far away from WE at the same side of the PET sheet (Figure 1). 

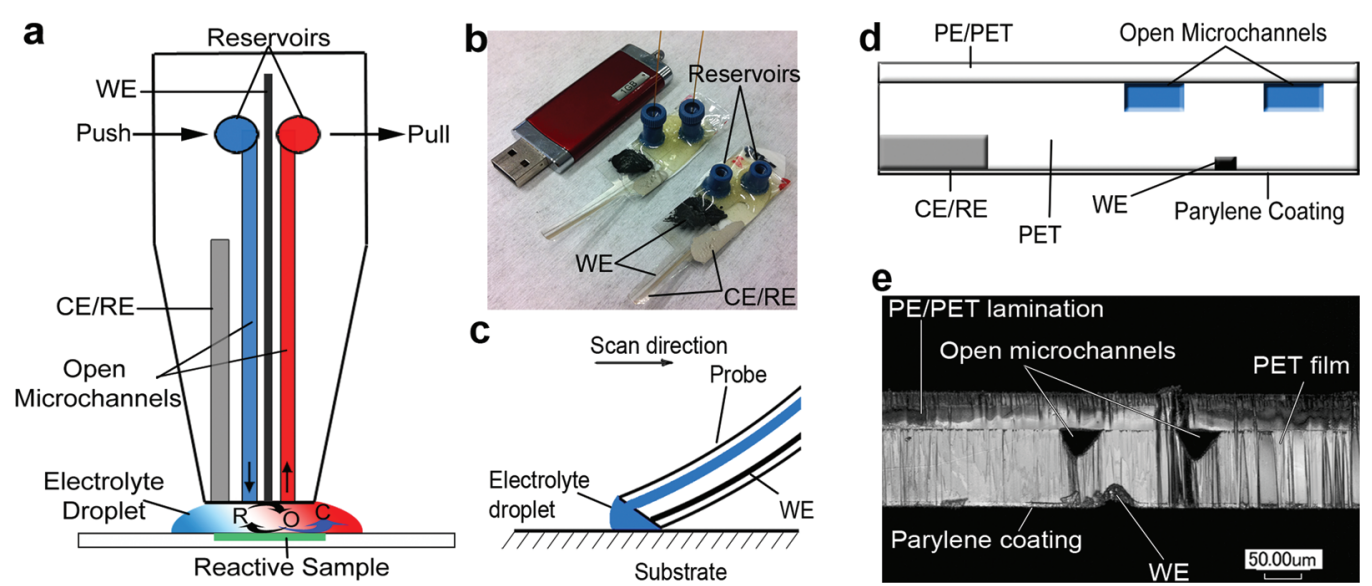

Figure 1. Push-pull probe concept. (a) General schematic representation; (b) optical photograph of two probes and a USB drive (to compare the size); (c) contact SECM mode for a push-pull probe; and arrangement of an exposed probe tip area (d) and microscope image (e).

After the tracks for WE and CE/RE were manually filled with carbon and $\mathrm{Ag} / \mathrm{AgCl}$ inks, respectively, and a curing step at $80^{\circ} \mathrm{C}$ for $1 \mathrm{~h}, \mathrm{PE} / \mathrm{PET}$ lamination was applied on the open microchannels side. The WE and CE/RE tracks side was then covered with a $5 \mu \mathrm{m}$ thick Parylene $\mathrm{C}$ film by use of a Parylene deposition system (Comelec SA, La Chaux-de-Fonds, Switzerland).

In order to connect the microfluidic system of the probe with an external pumping system (syringe pump, KD Scientific, 250 and $500 \mu \mathrm{L}$, Gastight syringes 1700 series, Hamilton) reservoirs (Nanoport Assembly, Upchurch Scientific) were attached to the probe with epoxy resin glue. The pump was connected with the microfluidic reservoirs via microcapillaries with internal diameter of $250 \mu \mathrm{m}$. In order to decrease the influence of convection, the pushing flow rate was set to $0.25 \mu \mathrm{L} \cdot \mathrm{min}^{-1}$. Effective suction of the liquid was achieved only at flow rates higher than $2.5 \mu \mathrm{L} \cdot \mathrm{min}^{-1}$, although in such case air is also aspirated.

A cross-section of the probe was then exposed by using laser ablation in order to improve hydrophilic properties of polymeric films or by mechanical blade cutting at a certain angle $\left(\approx 29^{\circ}\right.$, vide infra) in order to improve the probe sensitivity. The quality of the electrodes and the shape of the exposed area were inspected with a scanning laser microscope (VK 8700, Keyence).

SECM Measurements. SECM measurements were carried out with a custom-built SECM setup controlled by SECMx software $^{62}$ and comprising an IVIUM compactstat (IVIUM Technologies, The Netherlands) operating in a classical threeelectrode mode. Data analyses were carried out with MIRA software. ${ }^{63}$ The electrochemical cell comprises $\mathrm{Ag} / \mathrm{AgCl}$ as $\mathrm{CE} / \mathrm{RE}$ and a carbon track as WE. All potentials are reported with respect to the $\mathrm{Ag} / \mathrm{AgCl}$ quasi-reference electrode (QRE). The samples were mounted on the bottom of a flat cell construction and investigated at room temperature $\left(20 \pm 2{ }^{\circ} \mathrm{C}\right)$.

SECM images in contact regime were acquired by using a liftoff routine included in SECMx software. ${ }^{56,57}$ In order to control successfully the probe bending direction, the push-pull device was mounted on a custom-built SECM holder with a predefined inclination angle of $70^{\circ}$, which was determined as a good compromise between diminishing topographic artifacts while maintaining an acceptable current contrast. ${ }^{56}$ In order to decrease $d$ and improve the probe response, the push-pull probe was placed on the holder in such way that the PET side covered with $5 \mu \mathrm{m}$ thick Parylene coating faced the sample surface. SECM line scan on a vertical surface was implemented by turning the holder with a probe $90^{\circ}$ and pressing the probe against the vertical substrate.

Inspection of the nanodroplet stability during experiments on initially dry surfaces was carried out with a digital microscope video camera Proscope HR with lens type 400X.

Numerical Simulations. The FEM simulations were performed by use of the finite element software COMSOL Multiphysics (version 3.5a), operated on Linux Ubuntu 8.04 platform with a four-core Mac Pro $2.66 \mathrm{GHz}$ CPU and $9.8 \mathrm{~Gb}$ of RAM. The Fick's diffusion equations were solved in dimensional form for the given three-dimensional (3D) geometrical model of a soft probe without taking into account convective contribution to the total flux. The mesh was refined down to the value of $0.2 \mu \mathrm{m}$ at the edge of the working microelectrode exposed area.

Simulation of approach curves was carried out with the assumption of probe bending as changing the probe inclination angle $\alpha$ with respect to sample surface after the probe and substrate get into a contact. Herein, as in previous reports ${ }^{55-57}$ we correlate $d$ with a new vertical coordinate $h_{\mathrm{P}}$ through the following relationships:

$$
d= \begin{cases}h_{\mathrm{P}}+l \cos \alpha & h_{\mathrm{P}} \geq 0, \text { contactless mode } \\ l \cos \alpha & h_{\mathrm{P}}<0, \text { contact mode }\end{cases}
$$

$l$ specifies the Parylene coating thickness. Details of the FEM simulation procedure, geometrical parameters of the model and mesh, and the description of a new vertical coordinate $h_{\mathrm{P}}$ are given in the Supporting Information (see section SI-1).

\section{RESULTS AND DISCUSSION}

Probe Characterization. A schematic representation of the push-pull probe is presented in Figure 1a, where a twoelectrode cell configuration allows us to perform SECM experiments when the WE and the CE/RE are in contact with a renewed nanoliter droplet of redox mediator solution. The droplet size can be roughly estimated as the volume underneath the active electrode area in contact mode, giving a value of $\sim 7.5$ $\mathrm{nL}$ (see Supporting Information, section SI-2). By pushing and pulling the electrolyte at the bottom of the probe, one can sustain electrolyte concentration within the droplet, avoiding problems arising from solvent evaporation. Optical photographs 


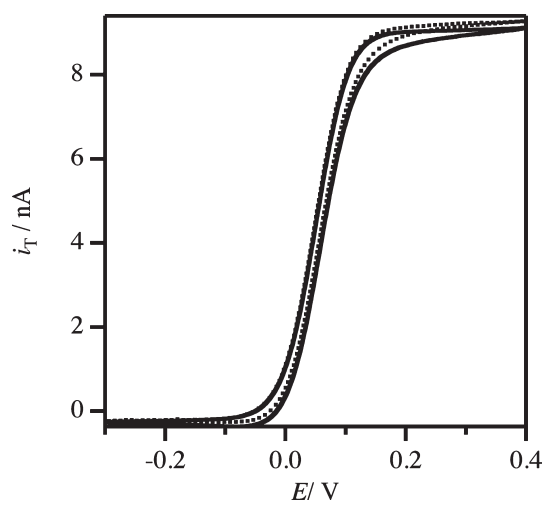

Figure 2. Cyclic voltammogram at a push-pull probe in the bulk of an aqueous solution containing $2.0 \mathrm{mM} \mathrm{FcCH}_{2} \mathrm{OH}$ and $0.1 \mathrm{M} \mathrm{KNO}_{3}$ with microfluidics on (dotted line) and off (solid line). Microfluidic flow rates of $0.2 \mu \mathrm{L} \cdot \mathrm{min}^{-1}$ for pushing and $2.5 \mu \mathrm{L} \cdot \mathrm{min}^{-1}$ for pulling liquid were employed. Scan rate was $20 \mathrm{mV} \cdot \mathrm{s}^{-1}$.

in Figure $1 \mathrm{~b}$ indicate the typical push-pull probe dimensions. The connection between the microfluidic channels and external pumps is established with the use of microfluidic reservoirs and electrical connection is performed at macroscopic contact pads. The push-pull probe is a robust, mechanically stable and versatile tool that can be easily operated in contact (see Figure 1c) or contactless SECM mode for surface reactivity investigations of initially dry surfaces or samples immersed under a thick electrolyte layer.

Figure $1 \mathrm{~d}$ reveals the configuration of an exposed active electrode area and depicts the relative position of the UME, the macroscopic $\mathrm{CE} / \mathrm{RE}$, and open microchannels. From the corresponding microscope image (Figure 1e) it is possible to observe the good parallel alignment between the triangularshaped open microchannels and the moon-shaped WE; the latter is located in the middle of the microfluidic system. Open microchannels for fluidics are enlarged in comparison with the size of the WE, since the open microchannel dimensions (i.e., size and shape) strongly affect the pressure gradients required for pushing/pulling liquids within a microstructure. ${ }^{64}$ Therefore, by increasing the microchannel size, one can avoid high pressures within the microfluidic system that can result in a delamination of the PE/PET film and subsequent probe destruction. Small probe-substrate working distances in SECM contact mode are achieved with the use of a thin $(\sim 5 \mu \mathrm{m})$ Parylene coating, providing additionally a mechanically and chemically stable pinhole-free insulation of the electrodes.

Electrochemical properties of push-pull probes were first characterized in contactless mode by cyclic voltammetry in a solution bulk of $2 \mathrm{mM} \mathrm{FcCH}_{2} \mathrm{OH}$ and $0.1 \mathrm{M} \mathrm{KNO}_{3}$ in order to avoid the influence from the substrate nature. The probe exhibits typical microelectrode behavior (Figure 2), that is, a steady-state diffusion-limited current with a relatively small contribution of capacitive current. It should be noted that, in the solution bulk, the influence of microfluidics on the probe response is almost negligible (dotted lines in Figure 2), indicating a minor contribution to the total flux of species by convection. However, as shown below, the key issue would be the influence of microfluidics in a contact mode when the probe response is affected in the vicinity of the substrate.

Sensitivity Improvement for Soft Probes. The recorded current contrast between active and inactive substrates in SECM can be improved by decreasing the probe-substrate distance $d$. As was previously reported, ${ }^{56}$ an increase of signal-to-noise ratio can be achieved when a soft probe coated with a thin Parylene film (e.g., $2-10 \mu \mathrm{m}$ ) is scanned in contact mode while the Parylene layer is facing the sample substrate. In addition to the mentioned Parylene coating, we report herein the increase of sensitivity in SECM measurements when the cross-sectional area of the probe is exposed by cutting with an optimized angle.

When the exposed area of the probe is perpendicular to the probe body and the probe physically touches the substrate (see Figure 3a), the smallest possible working distance $d$ is limited by the thickness of the polymeric coating (e.g., Parylene film) $l$ and the probe inclination angle $\alpha$ :

$$
d=l \sin \left(90^{\circ}-\alpha\right)
$$

When the cross-section of the probe is cut off at a certain angle $\beta$, the working distance diminishes:

$$
d=l \frac{\sin \left[90^{\circ}-(\alpha+\beta)\right]}{\cos \beta}=l \frac{\cos (\alpha+\beta)}{\cos \beta}
$$

In the present case (probe inclination angle $\alpha=70^{\circ}$ ), $d$ could be described as the linear function $d / l=0.345-0.017 \beta$ (a more general solution is given in Supporting Information section SI-3) and reaches 0 when $\beta$ approaches $20^{\circ}$. As expected, the current contrast, given as the normalized difference between current values at active and inactive substrates $\left(i_{\text {pos }}-i_{\text {neg }}\right) / i_{\mathrm{T}, \infty}$, improves with a decrease of $d$, in this case as a consequence of the used cutting angle $\beta$ (see Figure $3 \mathrm{~b}$ ). Of course, if $\alpha+\beta>90^{\circ}$, eq 3 is not valid and the minimal working distance that can be achieved is always 0 , where the highest current sensitivity is reached.

The effect of $\beta$ on the theoretical approach curve profiles is shown in Figure $3 c-$ e. In the presented example $\left(\beta \leq 20^{\circ}\right)$, the maximal sensitivity corresponds to the point where the active electrode area of the probe gets into a contact with the substrate. In such a situation, a further lowering of the probe (i.e., at negative $h_{\mathrm{p}}$ values) produces a decrease in the sensitivity as $d$ is increased when the probe bends more.

At large exposure angles $\left(\alpha+\beta>90^{\circ}\right.$ or $\beta>20^{\circ}$ for the presented example), it is very important to press the probe against the substrate, as a smaller working distance could be achieved after the soft probe touches the sample. Figure $3 \mathrm{e}$ shows the resulting simulated approach curve to an insulating and a conductive substrate for such situation. The experimental approach curves shown in Figure 4 resemble the behavior predicted by computations in Figure 3e. The difference between theoretical and experimental results appears most likely from a nonparallel alignment between the active electrode area and the substrate.

The influence of the convective fluxes on the approach curves at different probe-substrate distances and probe bending degrees is depicted in Figure 4. As mentioned above, the influence of microfluidics is almost negligible in the solution bulk. However, convection increases as the gap between tip and substrate diminishes. The resulting contribution from convective flux to the whole mass transport near the active electrode of the probe leads to a current increase when the probe is in proximity to the sample surface for both active and nonactive areas. Nevertheless, upon further probe bending, this effect is overcome as $d$ decreases. Therefore, the convective contribution to the overall current is almost negligible in a microdomain that is formed near the tip where the liquid that flows near the active area of the electrode is slowed down by increased friction. The previously mentioned effects of 

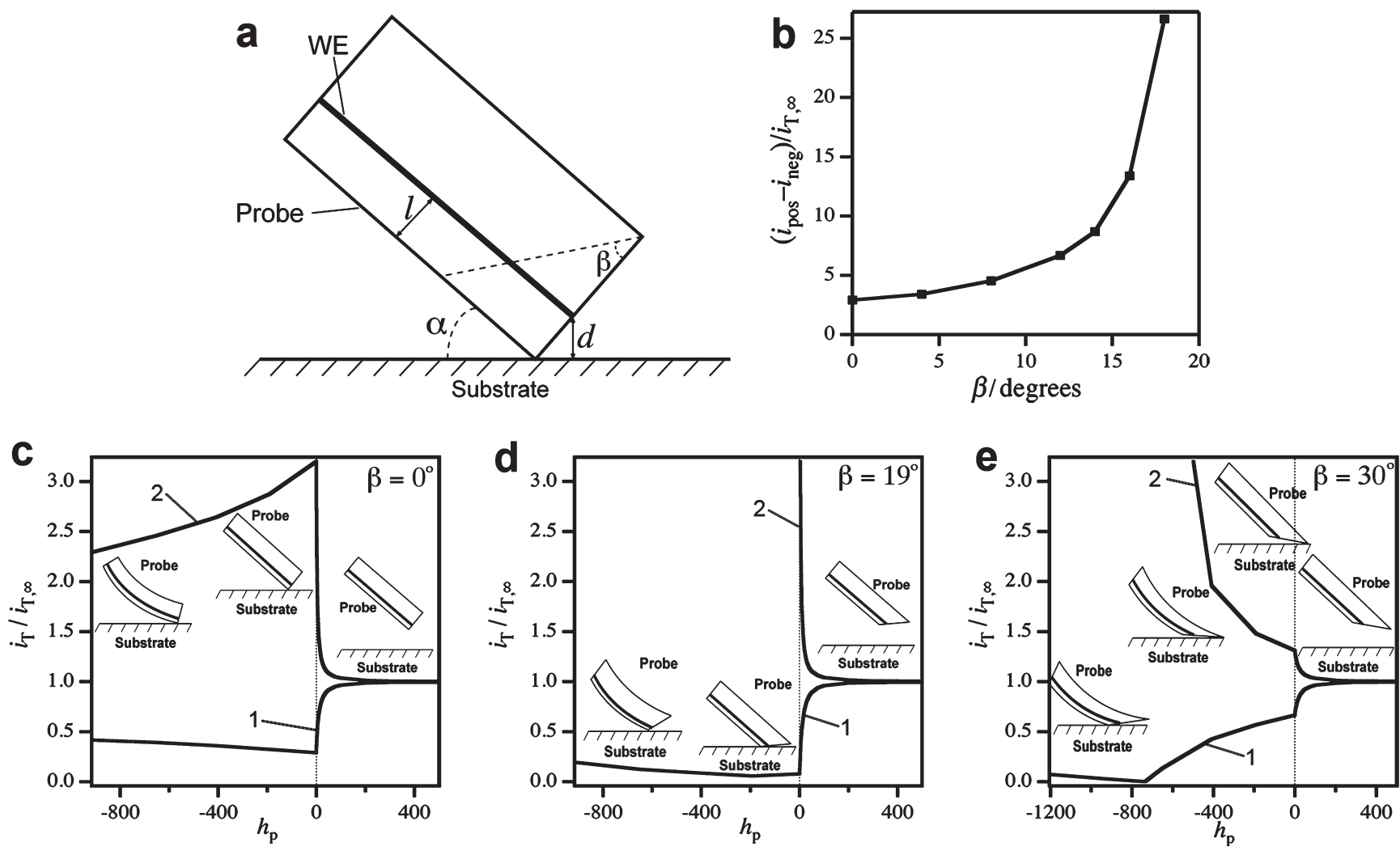

Figure 3. Sensitivity improvement for soft probes. (a) Schematic representation of the probe placed in a contact with the substrate. $d, l, \alpha$, and $\beta$ specify working distance, Parylene coating thickness, probe inclination, and exposure angle, respectively. (b) Normalized current contrast $\left(i_{\text {pos }}-i_{\text {neg }}\right) / i_{\mathrm{T}, \propto}$ as a function of the exposure angle $\beta$. (c-e) Simulated SECM feedback approach curves over an insulating (1) and a conductive (2) substrate for soft probes with a cross-sectional area exposed at $\beta$ equal to (c) $0^{\circ}$, (d) $19^{\circ}$, and (e) $30^{\circ}$. Insets depict the probe position and bending with respect to the substrate.

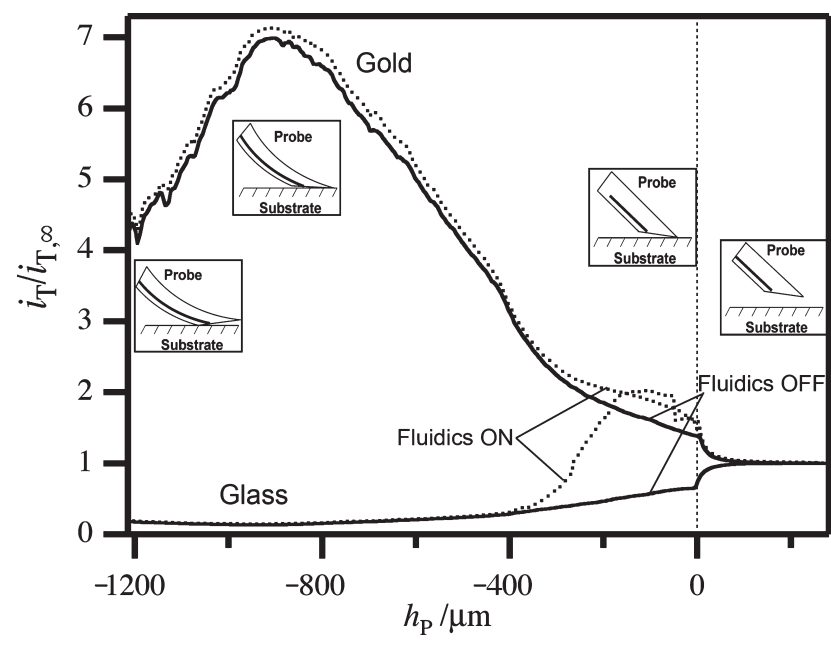

Figure 4. Experimental approach curves with push-pull probe (with cross-sectional area exposed at $\beta \approx 29^{\circ}$ ) over insulating glass and unbiased gold film in $2.0 \mathrm{mM} \mathrm{FcCH} \mathrm{FH}_{2} \mathrm{OH}$ and $0.1 \mathrm{M} \mathrm{KNO}_{3}$ aqueous solution with (dotted lines) and without (solid lines) microfluidics working. Tip potential $E_{\mathrm{T}}=0.3 \mathrm{~V}$, translation rate $\nu_{\mathrm{T}}=10 \mu \mathrm{m} \cdot \mathrm{s}^{-1}$.

microfluidics for push - pull probes exhibit a very similar behavior to the one presented by the recently reported fountain pen probes. ${ }^{57}$

SECM Measurements on Initially Dry Surfaces. In order to demonstrate the push-pull probe capabilities for surface activity characterization, an investigation of various gold-on-glass samples was carried out. In all experiments the interface remained dry during scanning, except a small region close to the probe tip that is in contact with the nanoliter droplet delivered by the microfluidic system. It is important to highlight that in the conditions in which the experiments were performed, no supporting electrolyte precipitation was found on the sample during imaging, even for experimental times as long as $6 \mathrm{~h}$.

Figure 5 exhibits the SECM images of various gold printed samples on a glass chip as the proof-of-concept of push-pull probe as a tool for surface reactivity characterization of initially dry systems. Thanks to the significant current contrast between conductive gold and insulating glass, the boundary between these two regions is clearly defined. The relative noise level in Figure 5 is related to the imbalanced flow rates for pushing and pulling electrolyte solution that causes changes in the droplet size (more details in Supporting Information section SI-4). In other words, the nanodroplet shrinks periodically. The changing droplet volume causes a pulsation of the amperometric signal. The faster the translation speed, the larger is the observed noise level. Despite this drawback, push-pull probes demonstrate good resolution for characterization of local surface reactivity as revealed in Figure 5a, where a SECM image of a gold printed electrode array of $50 \mu \mathrm{m}$ width gold bands with an interelectrode distance equal to $50 \mu \mathrm{m}$ is presented. The fact that the microchip structure is well-resolved and gold lines of different lengths are clearly distinguished demonstrates that the achieved resolution is high enough to precisely localize surface reactivity of micrometer dimensions. Push-pull probes also could be used for imaging with high lateral probe translation rates, as required, for instance, for large area 
a

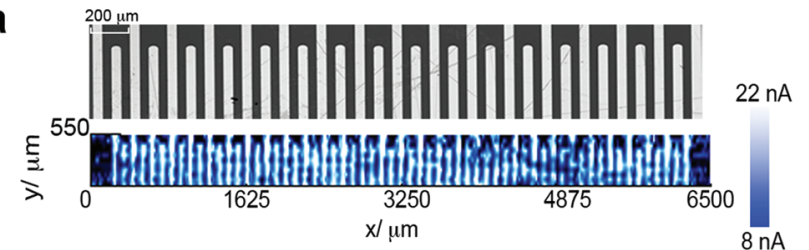

b

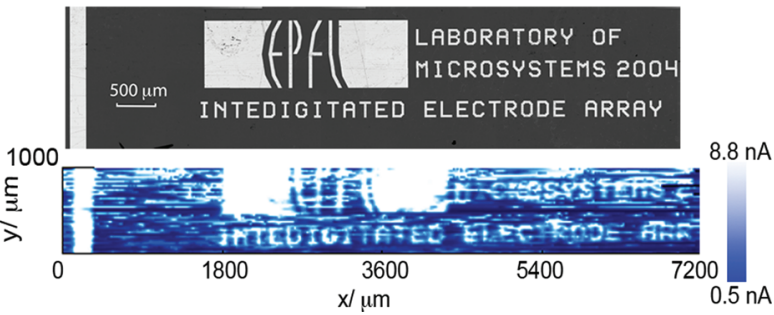

Figure 5. SECM imaging with push-pull probes. After each forward scan, the probe was retracted by a stroke height of $600-3000 \mu \mathrm{m}$ and placed back at the original horizontal $x$ position but with a shift of $50 \mu \mathrm{m}$ in the $y$ direction (Figure 5a) or $25 \mu \mathrm{m}$ (Figure $5 \mathrm{~b}$ ). All SECM images were acquired at $E_{\mathrm{T}}=0.3 \mathrm{~V}$ in $2.0 \mathrm{mM} \mathrm{FcCH}_{2} \mathrm{OH}$ and $0.1 \mathrm{M} \mathrm{KNO}_{3}$ aqueous solution with flow rates of $0.2 \mu \mathrm{L} \cdot \mathrm{min}^{-1}$ for pushing and $2.5 \mu \mathrm{L} \cdot \mathrm{min}^{-1}$ for pulling. (a) Optical photograph (top) and SECM image (bottom) of a gold printed interdigitated array of gold lines $50 \mu \mathrm{m}$ in width separated by $50 \mu \mathrm{m}$ interelectrode distance. Step size $=50 \mu \mathrm{m}$, $v_{\mathrm{T}}=20 \mu \mathrm{m} \cdot \mathrm{s}^{-1}$. (b) Optical (top) and SECM images (bottom) of a gold EPFL logo. $\nu_{\mathrm{T}}=200 \mu \mathrm{m} \cdot \mathrm{s}^{-1}$ with a step of $20 \mu \mathrm{m}$.

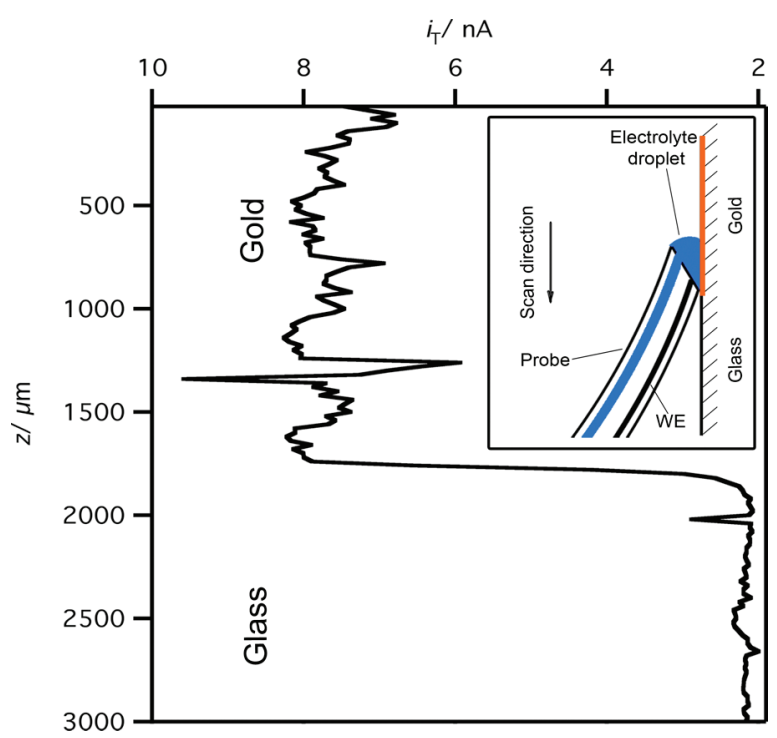

Figure 6. SECM line scan over a vertical gold/glass border taken with a push - pull probe at a translation speed $\nu_{\mathrm{T}}=50 \mu \mathrm{m} \cdot \mathrm{s}^{-1}$, with a step size $=$ $20 \mu \mathrm{m}$. The employed microfluidic push and pull flow rates of an aqueous solution containing $2.0 \mathrm{mM} \mathrm{FcCH}_{2} \mathrm{OH}$ and $0.1 \mathrm{M} \mathrm{KNO}_{3}$ were 0.2 and $2.5 \mu \mathrm{L} \cdot \mathrm{min}^{-1}$, respectively. $E_{\mathrm{T}}=0.3 \mathrm{~V}$. (Inset) $\mathrm{SECM}$ configuration during this experiment.

scans (Figure 5b). In such cases, the stability of the electrolyte droplet that should be held at the probe tip might be a key issue. As observed in Figure 5b, complex features such as a gold printed EPFL logo and microstructured text are adequately resolved even at a translation rate of $200 \mu \mathrm{m} \cdot \mathrm{s}^{-1}$ despite the large noise level.

SECM on a Vertical Surface. Investigation of initially dry samples that cannot be immersed into electrolyte solution might require the study of vertical surfaces. Such a configuration is not typical for SECM experiments but could be useful, for instance, in forensic sciences and corrosion inspections. The fact that an electrochemical cell is established within a nanodroplet of a redox mediator solution and the liquid is sustained by microfluidics and held by surface tension near the active electrode area justifies the possibility to use push-pull devices for SECM measurements on surfaces of any orientation with respect to gravitation force (i.e., tilted or vertical). The microfluidic push-pull probes are easily implemented for chemical activity detection over initially dry and vertically placed samples with a minimal change of SECM configuration, showing the versatility of this approach.

Figure 6 shows a SECM line scan performed with a push-pull probe on a vertically oriented gold-glass border by simply changing the orientation of the SECM holder. After the contact between the probe and the sample was established, the probe was pressed toward the substrate in order to achieve minimal working distances due to the probe bending (inset in Figure 6). Afterward, by scanning the probe in $z$ direction (i.e., parallel to the sample plane) while being in contact with the sample, the recorded amperometric signal showed clearly the capabilities of push-pull probes to resolve electrochemically active regions at vertically oriented interfaces within a renewed electrolyte nanodroplet.

\section{CONCLUSION}

The present push-pull probes demonstrate a new concept for mapping (electro)chemical information of initially dry samples with high spatial resolution. The possibilities to avoid immersing the sample under study into a thick layer of electrolyte solution and to investigate vertical or tilted substrates are the key advantages of the new push-pull probes. This is achieved by the use of microfluidics to sustain a nanodroplet of liquid in between the probe tip and the studied interface. As with other soft probes, push-pull is used in a contact regime, therefore the investigation of corrugated or curved samples is feasible, and the time-consuming procedure of sample leveling prior to SECM imaging could be evaded.

In addition, FEM simulations indicated how the contrast between active and nonactive sample areas is enlarged as a result of exposing the active electrode area at a certain angle. As confirmed by the experimental results, such improvement decreases significantly the contributions from convective fluxes to the amperometric signal of the probe.

All in all, the push-pull probe for SECM is a very promising tool that could prospectively be coupled to other analytical techniques such as mass spectrometry, high-performance liquid chromatography, or capillary electrophoresis. This opens the horizons for simultaneous chemical and electrochemical imaging for a wide range of sample surfaces. Following the approaches of Li et al. ${ }^{65}$ and Modestov et al., ${ }^{66}$ the future work will be focused on integrating push-pull with on- or off-line mass-spectrometric detection for imaging of local reactivity, monitoring products, byproducts, and (electro)chemical reaction intermediates with high spatial resolution, as well as for analytical purposes, like detection of explosives or narcotic drugs on human fingerprints.

\section{ASSOCIATED CONTENT}

Supporting Information. Additional text and six figures, showing details of FEM simulation procedure, geometrical parameters of computational model and mesh, description of vertical coordinate $h_{\mathrm{P}}$, and calculated dependence of probe-substrate 
distance $d$ on probe inclination angle $\alpha$ and angle of exposure $\beta$; as well as video files showing the nanodroplet during scanning. This material is available free of charge via the Internet at http:// pubs.acs.org

\section{AUTHOR INFORMATION}

\section{Corresponding Author}

*E-mail: hubert.girault@epfl.ch. Telephone: +41-21-693 3145. Fax: +41-21-693 3667.

\section{ACKNOWLEDGMENT}

This work was supported by SNCF Grants 20735001 and 20628506 and by a collaborative grant of SNF and Deutsche Forschungsgemeinschaft (SNF 20PA21_121570/1; DFG Wi $1617 / 10)$ "High throughput SECM imaging". We also acknowledge Jacques Josserand for assistance in FEM simulations and Valérie Devaud and Cyrille Hibert for technical support.

\section{REFERENCES}

(1) Bhushan, B.; Fuchs, H.; Tomitori, M. Applied scanning probe methods X: biomimetics and industrial applications; Springer: Heidelberg, Germany, 2008.

(2) Engstrom, R. C.; Weber, M.; Wunder, D. J.; Burgess, R.; Winquist, S. Anal. Chem. 1986, 58, 844-848.

(3) Barker, A. L.; Gonsalves, M.; MacPherson, J. V.; Slevin, C. J.; Unwin, P. R. Anal. Chim. Acta 1999, 385, 223-240.

(4) Bard, A. J.; Fan, F. R. F.; Pierce, D. T.; Unwin, P. R.; Wipf, D. O.; Zhou, F. Science 1991, 254, 68-74.

(5) Li, F.; Su, B.; Cortés-Salazar, F.; Nia, R. P.; Girault, H. H. Electrochem. Commun. 2009, 11, 473-476.

(6) Li, F.; Edwards, M.; Guo, J.; Unwin, P. R. J. Phys. Chem. C 2009, 113, 3553-3565.

(7) Ciani, I.; Burt, D. P.; Daniele, S.; Unwin, P. R. J. Phys. Chem. B 2004, 108, 3801-3809.

(8) Slevin, C. J.; Macpherson, J. V.; Unwin, P. R. J. Phys. Chem. B 1997, 101, 10851-10859.

(9) Wittstock, G.; Burchardt, M.; Pust, S. E.; Shen, Y.; Zhao, C. Angew. Chem., Int. Ed. 2007, 46, 1584-1617.

(10) Taylor, A. W.; Qiu, F. L.; Hu, J. P.; Licence, P.; Walsh, D. A. J. Phys. Chem. B 2008, 112, 13292-13299.

(11) Sun, P.; Zhang, Z. Q.; Gao, Z.; Shao, Y. H. Angew. Chem., Int. Ed. 2002, 41, 3445-3448.

(12) Slevin, C. J.; Ryley, S.; Walton, D. J.; Unwin, P. R. Langmuir 1998, 14, 5331-5334.

(13) Minguzzi, A.; Alpuche-Aviles, M. A.; Lopez, J. R.; Rondinini, S.; Bard, A. J. Anal. Chem. 2008, 80, 4055-4064.

(14) Jang, J. S.; Lee, J.; Ye, H.; Fan, F. R. F.; Bard, A. J. J. Phys. Chem. C 2009, 113, 6719-6724.

(15) Eckhard, K.; Erichsen, T.; Stratmann, M.; Schuhmann, W. Chem.-Eur. J. 2008, 14, 3968-3976.

(16) Lee, J. W.; Ye, H. C.; Pan, S. L.; Bard, A. J. Anal. Chem. 2008, 80, 7445-7450.

(17) Gyurcsanyi, R. E.; Jagerszki, G.; Kiss, G.; Toth, K. Bioelectrochemistry 2004, 63, 207-215.

(18) Basame, S. B.; White, H. S. J. Phys. Chem. 1995, 99, 16430-16435.

(19) Bard, A. J.; Mirkin, M. V.; Unwin, P. R.; Wipf, D. O. J. Phys. Chem. 1992, 96, 1861-1868.

(20) Lefrou, C.; Cornut, R. ChemPhysChem 2010, 11, 547-556.

(21) Cornut, R.; Hapiot, P.; Lefrou, C. J. Electroanal. Chem. 2009, 633, 221-227.

(22) Sanchez-Sanchez, C. M.; Rodiriguez-Lopez, J.; Bard, A. J. Anal. Chem. 2008, 80, 3254-3260.
(23) Rodriguez-Lopez, J.; Bard, A. J. J. Am. Chem. Soc. 2010, 132, 5121-5129.

(24) Zoski, C. G.; Mirkin, M. V. Anal. Chem. 2002, 74, 1986-1992.

(25) Pust, S. E.; Salomo, M.; Oesterschulze, E.; Wittstock, G. Nanotechnology 2010, 21, No. 105709.

(26) Fan, F. R. F.; Bard, A. J. Science 1995, 270, 1849-1851.

(27) Forouzan, F.; Bard, A. J. J. Phys. Chem. B 1997, 101, 10876-10879.

(28) Hassel, A. W.; Lohrengel, M. M. Electrochim. Acta 1997, 42, $3327-3333$.

(29) Lohrengel, M. M.; Moehring, A.; Pilaski, M. Fresenius' J. Anal. Chem. 2000, 367, 334-339.

(30) Lohrengel, M. M.; Moehring, A.; Pilaski, M. Electrochim. Acta 2001, 47, 137-141.

(31) Williams, C. G.; Edwards, M. A.; Colley, A. L.; Macpherson, J. V.; Unwin, P. R. Anal. Chem. 2009, 81, 2486-2495.

(32) Ebejer, N.; Schnippering, M.; Colburn, A. W.; Edwards, M. A.; Unwin, P. R. Anal. Chem. 2010, 82, 9141-9145.

(33) Spaine, T. W.; Baur, J. E. Anal. Chem. 2001, 73, 930-938.

(34) Turcu, F.; Schulte, A.; Schuhmann, W. Anal. Bioanal. Chem. 2004, 380, 736-741.

(35) Day, T. M.; Unwin, P. R.; Macpherson, J. V. Nano Lett. 2007, 7, $51-57$

(36) Suryavanshi, A. P.; Yu, M.-F. Nanotechnology 2007, 18, No. 105305.

(37) Ball, J. C.; Scott, D. L.; Lumpp, J. K.; Daunert, S.; Wang, J.; Bachas, L. G. Anal. Chem. 2000, 72, 497-501.

(38) Bratten, C. D. T.; Cobbold, P. H.; Cooper, J. M. Anal. Chem. $1997,69,253-258$.

(39) Ludwig, M.; Kranz, C.; Schuhmann, W.; Gaub, H. E. Rev. Sci. Instrum. 1995, 66, 2857-2860.

(40) Hengstenberg, A.; Kranz, C.; Schuhmann, W. Chem.-Eur. J. 2000, 6, 1547-1554.

(41) Katemann, B. B.; Schulte, A.; Schuhmann, W. Chem.-Eur. J. 2003, 9, 2025-2033.

(42) Lee, Y.; Ding, Z. F.; Bard, A. J. Anal. Chem. 2002, 74, 3634-3643.

(43) James, P. I.; Garfias-Mesias, L. F.; Moyer, P. J.; Smyrl, W. H. J. Electrochem. Soc. 1998, 145, L64-L66.

(44) Comstock, D. J.; Elam, J. W.; Pellin, M. J.; Hersam, M. C. Anal. Chem. 2010, 82, 1270-1276.

(45) Takahashi, Y.; Shevchuk, A. I.; Novak, P.; Murakami, Y.; Shiku, H.; Korchev, Y. E.; Matsue, T. J. Am. Chem. Soc. 2010, 132, 10118-10126.

(46) Kurulugama, R. T.; Wipf, D. O.; Takacs, S. A.; Pongmayteegul, S.; Garris, P. A.; Baur, J. E. Anal. Chem. 2005, 77, 1111-1117.

(47) Alpuche-Aviles, M. A.; Wipf, D. O. Anal. Chem. 2001, 73, 4873-4881.

(48) McKelvey, K.; Edwards, M. A.; Unwin, P. R. Anal. Chem. 2010, 82, 6334-6337.

(49) Wang, L. Q.; Kranz, C.; Mizaikoff, B. Anal. Chem. 2010, 82, 3132-3138.

(50) Macpherson, J. V.; Unwin, P. R. Anal. Chem. 2000, 72, 276-285.

(51) Jones, C. E.; Macpherson, J. V.; Barber, Z. H.; Somekh, R. E.; Unwin, P. R. Electrochem. Commun. 1999, 1, 55-60.

(52) Kueng, A.; Kranz, C.; Lugstein, A.; Bertagnolli, E.; Mizaikoff, B. Angew. Chem., Int. Ed. 2003, 42, 3238-3240.

(53) Sklyar, O.; Kueng, A.; Kranz, C.; Mizaikoff, B.; Lugstein, A.; Bertagnolli, E.; Wittstock, G. Anal. Chem. 2005, 77, 764-771.

(54) Salomo, M.; Pust, S. E.; Wittstock, G.; Oesterschulze, E. Microelectron. Eng. 2010, 87, 1537-1539.

(55) Cortes-Salazar, F.; Träuble, M.; Li, F.; Busnel, J. M.; Gassner, A. L.; Hojeij, M.; Wittstock, G.; Girault, H. H. Anal. Chem. 2009, $81,6889-6896$.

(56) Cortes-Salazar, F.; Momotenko, D.; Lesch, A.; Wittstock, G.; Girault, H. H. Anal. Chem. 2010, 82, 10037-10044.

(57) Cortes-Salazar, F.; Lesch, A.; Momotenko, D.; Busnel, J. M.; Wittstock, G.; Girault, H. H. Anal. Methods 2010, 2, 817-823. 
(58) Juncker, D.; Schmid, H.; Delamarche, E. Nat. Mater. 2005, 4, 622-628.

(59) Kaigala, G. V.; Lovchik, R. D.; Drechsler, U.; Delamarche, E. Langmuir 2011, 27, 5686-5693.

(60) Roberts, M. A.; Rossier, J. S.; Bercier, P.; Girault, H. H. Anal. Chem. 1997, 69, 2035-2042.

(61) Rossier, J. S.; Bercier, P.; Schwarz, A.; Loridant, S.; Girault, H. H. Langmuir 1999, 15, 5173-5178.

(62) Nunes, K.; Hallmeier, K.; Szargan, R.; Raschke, T.; Radehaus, C.; Wittstock, G. Electroanalysis 2007, 19, 1023-1031.

(63) Wittstock, G.; Asmus, T.; Wilhelm, T. Fresenius' J. Anal. Chem. 2000, 367, 346-351.

(64) Nguyen, N.-T.; Wereley, S. T. Fundamentals and applications of microfluidics; Artech House: Boston, MA, 2002.

(65) Li, N.; Eckhard, K.; Assmann, J.; Hagen, V.; Otto, H.; Chen, X. X.; Schuhmann, W.; Muhler, M. Rev. Sci. Instrum. 2006, 77, No. 084102.

(66) Modestov, A. D.; Srebnik, S.; Lev, O.; Gun, J. Anal. Chem. 2001, 73, 4229-4240. 